\title{
Hybrid Peer-to-peer Streaming System for Mobile Peers with Transcoding
}

\author{
Shuai Zeng \\ Guo-feng Zhao \\ Chuan Xu \\ Institute of Future Internet Technology Institute of Future Internet Technology Institute of Future Internet Technology \\ Chongqing University of Posts and \\ Telecommunications \\ Chongqing, P. R. China, 400065 \\ maillinger@gmail.com \\ Chongqing University of Posts and \\ Telecommunications \\ Chongqing, P. R. China, 400065 \\ zhaogf@cqupt.edu.cn \\ Chongqing University of Posts and \\ Telecommunications \\ Chongqing, P. R. China, 400065 \\ xuchuan@cqupt.edu.cn
}

\begin{abstract}
In this paper, we study in hybrid peer-to-peer (P2P) streaming system, which includes fixed peers and mobile peers in the same network, and propose a flow rate allocation algorithm to optimize the streaming system. In the proposed algorithm, based on transcoding technique, we describe how to exchange original and transcoded data among video source server, fixed and mobile peers. The purpose of our algorithm is to reduce the bandwidth demand of video source server, while ensuring the flow rate of video data sending to any peer no less than its video coding rate. We compare the performance of P2P streaming system using our algorithm with traditional design in various situations in the simulation experiment, and test how much benefit the system can get from the new algorithm. The results show that, if the flow rate of video data is allocated appropriately, better performance of streaming system can be achieved.
\end{abstract}

\section{Keywords}

Streaming system, peer-to-peer network, video transmission, data distribution, mobile communications.

\section{INTRODUCTION}

With the popular of P2P multimedia streaming, peers become more and more complex. These clients, such as PC, TV, tablet, PDA, cellphone, and so on, have various screen sizes, color depth and video qualities. Specially, they may have different bandwidth with heterogeneous access network [1-4], and they may have different video coding algorithm with heterogeneous hardware and software. In traditional designs, the peers that receive different video coding data cannot share their upload bandwidth each other.

To deal with this problem, in recent years, there are some literatures [2-6] that propose transcoding technique using in P2P streaming systems. With this technique, powerful peers can transcode and share their video data to other peers, which even use different video coding algorithm. Present researches announce that, in some situations, compared with traditional design, P2P streaming system with transcoding have better performances, such as suitability of heterogeneous drivers, various video coding support, and less resource consumption. Nevertheless, existent studies just focus on video coding algorithm and computational resource allocation, which fails to investigate and design their systems on how to efficiently transmit different video coding data in the network.

In this paper, we are interested in the algorithm of flow rate allocation. Prior works have studied the issue of flow rate allocation in no transcoding situation [7-9], the scenario of network coding [10], the multiple channel system [11], and the arbitrary graph model [12]. Our study bases on hybrid P2P streaming system with transcoding, which includes fixed and mobile peers in one overlay network [4][7]. Fixed peers, in this study, refer to PC or IPTV connected to the overlay network over a reliable connection and had powerful computing capability to transcode video data timely. And oppositely, mobile peers are seen as no transcoding capability, but just transmit transcoded data. We seek to propose a flow rate allocation algorithm for transcoding and transmission of video streaming to mobile peers, while ensure the flow rate of video data sending to any peer no less than its video coding rate. Without sacrificing realistic assumptions of systems scale, our analysis and algorithm is based on both transcoding technique and the feature of hybrid network environment, and we mainly investigate the bandwidth demand of video source server in the system using our algorithm. Furthermore, based on the simulation experiment, we compare the performance of our design with traditional situations, and seek to find out in what situation our algorithm work more effectively and how much benefit the system can get.

The remainder of the paper is organized as follows. Section 2 describes and insights the basic environment of research. In Section 3, we give the flow rate allocation algorithm of the hybrid system for streaming to mobile peers. In section 4 , we show the simulation experiment and compare system performance with traditional design. Finally, we conclude this paper in Section 5.

\section{HYBRID P2P STREAMING SYSTEM WITH TRANSCODING}

Before we propose our flow rate allocation algorithm, in this section, we first summarize some fundamental characteristics and overview the research environment of hybrid P2P streaming system with transcoding (HPSST). 
Utilizing transcoding technique, hybrid P2P streaming system can support multiple video coding rates and exchange their data in one overlay network. Especially, in our study, there are two kinds of video coding data needed by fixed and mobile peers respectively. As shown as Fig. 1, this is a simple example of HPSST.

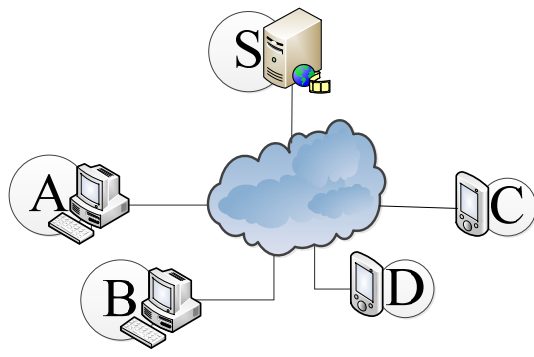

Figure 1. Example of HPSST.

There are four peers (A, B, C and D) and one video source server (S) in the system. A and B are PCs with $1000 \mathrm{Kbps}$ upload capacity and $800 \mathrm{Kbps}$ video coding rate respectively, which are seen as fixed peers, and C, D are cellphones with $100 \mathrm{Kbps}$ upload capacity and 200Kbps video coding rate respectively, which are seen as mobile peers. And, we further assume that the download bandwidth and transcoding capability is not the bottleneck of the system. The goal of flow rate allocation is to ensure all peers receiving data achieving their video coding rate, and, at the same time, minimize the bandwidth demand of video source server. Fig. 2(a) and Fig. 2(b) are two flow rate allocation methods. They all ensure receiving flow rate of fixed peers at $800 \mathrm{Kbps}$ and mobile peers at 200Kbps. But the demand bandwidth of video source server is $1100 \mathrm{Kbps}$ in Fig. 2(a), while 900Kbps in Fig. 2(b).

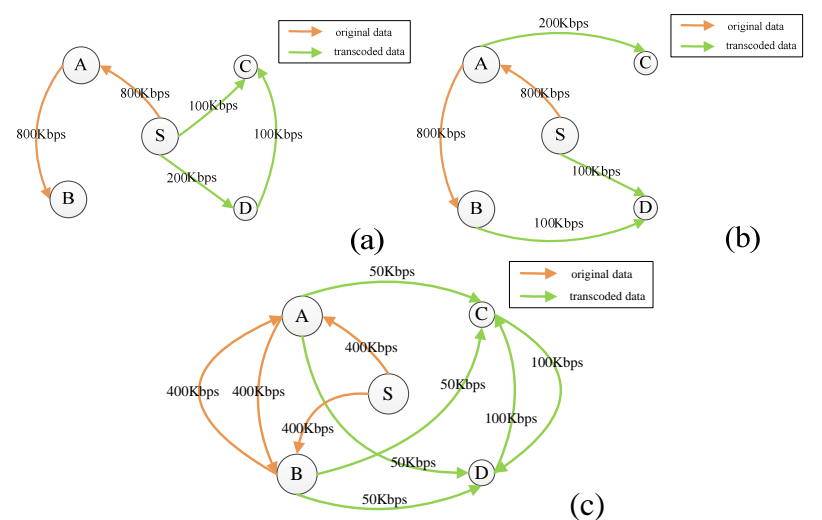

Figure 2. Examples of flow rate allocation in HPSST.

As we see in the examples of Fig. 2, the flow rate allocation influences the performance of the system. In next section, we describe how our algorithm works. Before that, for the sake of mathematical tractability, we make a few assumptions. First, our study bases on the scenario of hybrid P2P streaming system. The all fixed peers are supposed to have the same upload capacity and video coding rate. And, the all mobile peers are supposed to have the same upload capacity and video coding rate too. Second, the connection of whole system is seen as a complete network, and the capacity of upload bandwidth is the only bottleneck. This assumption is according to most existing studies [7-10] of P2P systems. Specially, in our study, it means that the fixed peers can transcode their video data to the format for mobile peers, and the transcoded data is always enough for upload in the discussion. Our flow rate allocation algorithm just discusses the basic data flow model and seeks to optimize the bandwidth utilization, which does not restrict the specific video coding algorithm. FLOW RATE ALLOCATION ALGORITHM

Before give our algorithm, introduce some necessary notations firstly.

Denote by $s$ for the video source server, $p_{i}^{f}$ for the i-th fixed peer, and $p_{i}^{m}$ for the i-th mobile peer. Let $n_{f}$ be the number of fixed peers and $n_{m}$ be the number of mobile peers. Let $u_{s}$ be the bandwidth demand of $s, u_{f}$ be the upload capacity of fixed peer, and $u_{m}$ be the upload capacity of mobile peer. Let $r_{o}$ be the coding rate of original video data and $r_{t}$ be the coding rate of transcoded video data. Let $t r_{i}^{f}$ be the total rate of $p_{i}^{f}$ getting video data from the system and $\operatorname{tr}_{i}^{m}$ be the total rate of $p_{i}^{m}$ getting video data from the system.

Denote by $f_{o}(x \rightarrow y)=z$ for network flow of original data from peer $\mathrm{x}$ (or video source server) to peer $\mathrm{y}$ at rate $\mathrm{z}$, and $f_{t}(x \rightarrow y)=z$ for network flow of transcoded data from peer $\mathrm{x}$ (or video source server) to peer y at rate z. $f_{o}(x \rightarrow y)=z$ and $f_{t}(x \rightarrow y)=z$ are the key parameters of the algorithm, which describe how data transmits in the system.

\section{The Flow of Original Data}

1) Get information of system, including $n_{f}, u_{f}, r_{o}, n_{m}$, $u_{m}$, and $r_{t}$;

2)If $\frac{\left(n_{f}-1\right) \cdot r_{o}}{n_{f}}-u_{f}>0$, go to 6$)$;

3)Let $u_{s}=r_{o}$, and allocate flow rate as follows:

a) $\quad f_{o}\left(s \rightarrow p_{i}^{f}\right)=\frac{r_{o}}{n_{f}}, \quad\left(\right.$ for $\left.i=1,2, \ldots, n_{f}\right)$;

b)

$$
\begin{aligned}
& f_{o}\left(p_{j}^{f} \rightarrow p_{i}^{f}\right)=\frac{r_{o}}{n_{f}}, \\
& \left(\text { for } i=1,2, \ldots, n_{f} ; j=1,2, \ldots, n_{f} ; i \neq j\right)
\end{aligned}
$$

4) $p_{i}^{f}$ receives original data at rate

$\operatorname{tr}_{i}^{f}=\frac{r_{o}}{n_{f}}+\frac{\left(n_{f}-1\right) \cdot r_{o}}{n_{f}}=r_{o}, \quad\left(\right.$ for $\left.i=1,2, \ldots, n_{f}\right) ;$

5) Go to 9);

6) Let $u_{s}=r_{t}+n_{f} \cdot\left(r_{o}-u_{f}\right)$, and allocate flow rate as follows:

a) $\quad f_{o}\left(s \rightarrow p_{i}^{f}\right)=\frac{u_{f}}{n_{f}-1}, \quad\left(\right.$ for $\left.i=1,2, \ldots, n_{f}\right)$;

b) $f_{o}\left(p_{j}^{f} \rightarrow p_{i}^{f}\right)=\frac{u_{f}}{n_{f}-1}$,

$\left(\right.$ for $\left.i=1,2, \ldots, n_{f} ; j=1,2, \ldots, n_{f} ; i \neq j\right)$ 
c) $\quad f_{o}\left(s \rightarrow p_{i}^{f}\right)=r_{o}-\frac{n_{f} \cdot u_{f}}{n_{f}-1}, \quad\left(\right.$ for $\left.i=1,2, \ldots, n_{f}\right)$;

7) $p_{i}^{f}$ receives original data at rate

$t r_{i}^{f}=\frac{u_{f}}{n_{f}-1}+\frac{\left(n_{f}-1\right) \cdot u_{f}}{n_{f}-1}+\left(r_{o}-\frac{n_{f} \cdot u_{f}}{n_{f}-1}\right)=r_{o}$,

( for $i=1,2, \ldots, n_{f}$ )

8) Go to 9);

The Flow of Transcoded Data

Let $u_{s}^{\prime}$ be the rest upload capacity of $s$, and $u^{\prime}{ }_{f i}$ be the rest upload capacity of $p_{i}^{f}$.

9) If $\frac{\left(n_{m}-1\right) \cdot r_{t}}{n_{m}}-u_{m}>0$, go to 14$)$;

10) Figure up $u^{\prime}{ }_{s}$ and $u^{\prime}{ }_{f i}$;

11) Allocate flow rate as follows:

a)

$$
f_{t}\left(s \rightarrow p_{i}^{m}\right)=\frac{r_{t} \cdot u_{s}^{\prime}}{n_{m} \cdot\left(u_{s}+n_{f} \cdot\left(u_{f}-r_{o}\right)\right)},
$$

( for $\left.i=1,2, \ldots, n_{m}\right)$

b)

$$
f_{t}\left(p_{j}^{f} \rightarrow p_{i}^{m}\right)=\frac{r_{t} \cdot u_{f j}^{\prime}}{n_{m} \cdot\left(u_{s}+n_{f} \cdot\left(u_{f}-r_{o}\right)\right)},
$$

( for $\left.i=1,2, \ldots, n_{m} ; j=1,2, \ldots, n_{f}\right)$

c)

$$
f_{t}\left(p_{j}^{m} \rightarrow p_{i}^{m}\right)=\frac{r_{t}}{n_{m}},
$$

$\left(\right.$ for $\left.i=1,2, \ldots, n_{m} ; j=1,2, \ldots, n_{m} ; i \neq j\right)$

12) $p_{i}^{m}$ receives transcoded data at rate

$t r_{i}^{m}=r_{t}, \quad\left(\right.$ for $\left.i=1,2, \ldots, n_{m}\right)$;

13) End.

14) Let $u_{s}=n_{f} \cdot\left(r_{o}-u_{f}\right)+n_{m} \cdot\left(r_{t}-u_{m}\right)$, and figure up $u^{\prime}{ }_{s}$ and $u^{\prime}{ }_{f i}$;

15) Allocate flow rate as follows:

a)

$$
f_{t}\left(s \rightarrow p_{i}^{m}\right)=\frac{u_{m} \cdot u_{s}^{\prime}}{\left(n_{m}-1\right) \cdot\left(u_{s}+n_{f} \cdot\left(u_{f}-r_{o}\right)\right)},
$$

( for $i=1,2, \ldots, n_{m}$ )

b)

$$
f_{t}\left(p_{j}^{f} \rightarrow p_{i}^{m}\right)=\frac{u_{m} \cdot u_{f j}^{\prime}}{\left(n_{m}-1\right) \cdot\left(u_{s}+n_{f} \cdot\left(u_{f}-r_{o}\right)\right)},
$$

$\left(\right.$ for $\left.i=1,2, \ldots, n_{m} ; j=1,2, \ldots, n_{f}\right)$

c)

$$
f_{t}\left(p_{j}^{m} \rightarrow p_{i}^{m}\right)=\frac{u_{m}}{n_{m}-1},
$$

$\left(\right.$ for $\left.i=1,2, \ldots, n_{m} ; j=1,2, \ldots, n_{m} ; i \neq j\right)$

d)

$$
f_{t}\left(s \rightarrow p_{i}^{m}\right)=\left(r_{t}-\frac{n_{m} \cdot u_{m}}{n_{m}-1}\right) \cdot\left(\frac{u_{s}^{\prime}}{u_{s}+n_{f} \cdot\left(u_{f}-r_{o}\right)}\right) \text {; }
$$

( for $\left.i=1,2, \ldots, n_{m}\right)$ e)

$$
\begin{aligned}
& f_{t}\left(p_{j}^{f} \rightarrow p_{i}^{m}\right)=\left(r_{t}-\frac{n_{m} \cdot u_{m}}{n_{m}-1}\right) \cdot\left(\frac{u_{f j}^{\prime}}{u_{s}+n_{f} \cdot\left(u_{f}-r_{o}\right)}\right), \\
& \left(\text { for } i=1,2, \ldots, n_{m} ; j=1,2, \ldots, n_{f}\right)
\end{aligned}
$$

16) $p_{i}^{m}$ receives transcoded data at rate

$$
\operatorname{tr}_{i}^{m}=r_{t}, \quad\left(\text { for } i=1,2, \ldots, n_{m}\right) \text {; }
$$

17) End.

Return to the HPSST example of Fig. 1. Following our algorithm, the flow rate allocation should be assigned as Fig. 2(c). Hence the bandwidth demand of video source server reduces to $800 \mathrm{Kbps}$, while all peers getting data are no less than their video coding rate. For more details, in next section, we do simulation experiment to test the effectiveness of our flow rate allocation algorithm.

\section{SIMULATION EXPERIMENT}

In the simulation experiment, we implement hybrid P2P streaming system with transcoding and traditional no transcoding design described in previous paper [8][9]. To conduct rigorous analysis of the systems under wide range of working conditions, we implement our experiments to emulate the characteristics of realistic systems with different parameters and a large number of test times. We mainly investigate the bandwidth demand of video source server using our algorithm, and compare the performance with traditional design in various settings. For brevity, denote by $u_{s}^{T}$ for the bandwidth demand of video source server in the simulation system using traditional algorithm, and by $u_{s}$ for our algorithm.

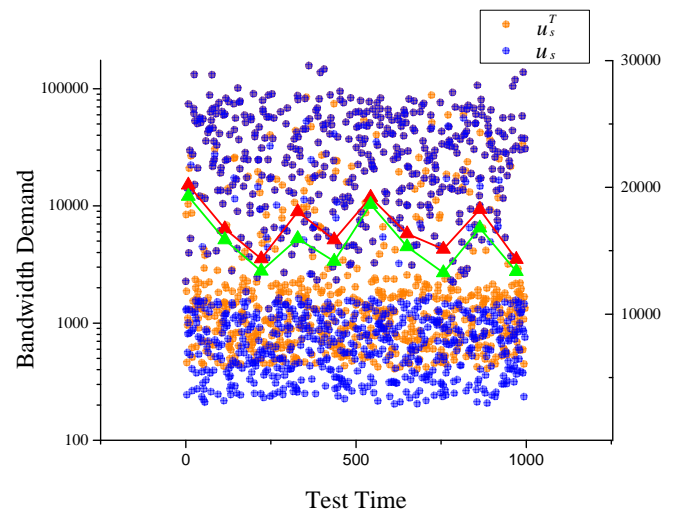

Figure 3. Random tests.

First, we take an overlook for the $u_{s}^{T}$ and $u_{s}$ in complete random scenario. The experiment tests the system with all random settings, which randomly chooses $n_{f}$ and $n_{m}$ from 5 to 100, $r_{o}$ and $r_{t}$ from 200 to $1600, u_{f}$ and $u_{m}$ from 100 to 2000. We test $u_{s}^{T}$ and $u_{s} 1000$ times respectively. The results are shown in Fig. 3. We can see that, in the complete random scenario, $u_{s}^{T}$ and $u_{s}$ spread all over the figure, but, notice of the lower part of Fig. 3, $u_{s}^{T}$ is hardly less than 400 , while $u_{s}$ descends to 200 in some cases. Furthermore, we count the averages of every 100 values of $u_{s}^{T}$, which are drawn with red triangles in Fig. 3, and the averages of $u_{s}$ are drawn with green. The new algorithm shows some advantages in this test.

Next, for more details, we initialize and test the system with practical parameter, which simulates the HPSST in ADSL and 
CDMA networks. ADSL clients are seen as the fixed peers, while CDMA clients are seen as the mobile peers. The initial setting of the HPSST is on Table I. In this test, we seek to know how the bandwidth demand of video source server changes by the different system scale. The peer number of ADSL and CDMA are both 20 initially. We add peer number in only one kind client every time, and measure the corresponding bandwidth demand of video source server. For example, as the green area in the bottom of Fig. 4, first, we measure the bandwidth demand of video source server with our algorithm in the initial setting as TABLE I, after that, peer number of ADSL is added to 30,40 , and so on, while other parameters are held constant, and we measure the bandwidth demand of video source server orderly. In the experiment, we compare the bandwidth demand of video source server with traditional and our algorithm from the increase of both ADSL and CDMA scales. The results are shown in Fig. 4. We can see that the bandwidth demand of video source server using our algorithm is less than traditional one both in the increase of ADSL and CDMA peers.

Table 1. Initial setting of HPSST

\begin{tabular}{|c|c|c|c|}
\hline Type & Capacity & Peer Number & Coding Rate \\
\hline ADSL & $1 \mathrm{Mbps}$ & 20 & 500Kbps \\
\hline CDMA & $100 \mathrm{Kbps}$ & 20 & $200 \mathrm{Kbps}$ \\
\hline
\end{tabular}

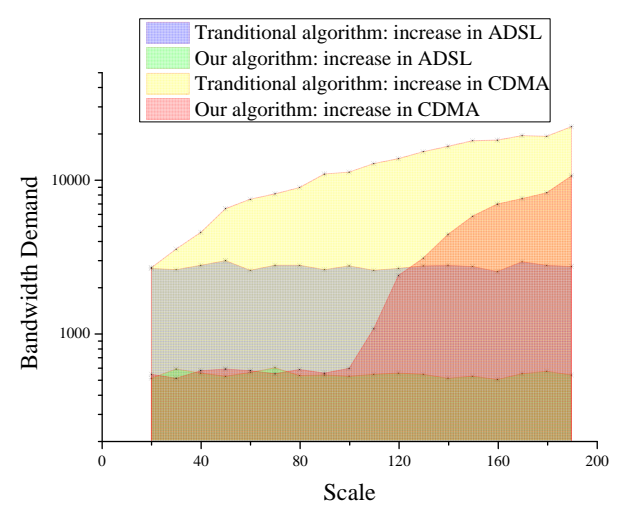

Figure 4. Bandwidth demand of video source server.

\section{CONCLUSION}

In this paper, we have studied the issue of streaming to mobile peers in hybrid P2P streaming system. With transcoding technique, we propose a flow rate allocation algorithm to optimize the system performance. We have also done simulation experiment to compare our algorithm to traditional design, and to test how much benefit new algorithm takes in various situations. The results have shown some advantages of our algorithm.

\section{ACKNOWLEDGMENTS}

This work is partially supported by The National Basic Research Program of China (973 Program) (2012CB315800), and The National Natural Science Foundation of China (61001084, $61402065)$. The authors are grateful to the anonymous referee for a careful checking of the details and for helpful comments that improved this paper.

\section{REFERENCES}

[1] T. Wolf, In-network service for customization in next generation networks. IEEE Network, vol. 24, no. 4, August 2010, pp. 6-12

[2] F. Chen, T. Repantis, and V. Kalogeraki, Coordinated media streaming and transcoding in peer-to-peer systems. In Proc. IEEE Int. Parallel Distributed Process. Symp., Apr. 2005, p. $56 b$.

[3] D. Liu, E. Setton, B. Shen, and S. Chen, PAT: Peer-assisted transcoding for overlay streaming to heterogeneous devices. In Proc. Int. Workshop Netw. Oper. Syst. Support Dig. Audio Video, Jun. 2007.

[4] J. Noh, M. Makar, and B. Girod. Streaming to mobile users in a peer-to-peer network. In International Mobile Multimedia Communications Conference, London, England, Sep. 2009.

[5] Zixia Huang, Chao Mei, Li Erran Li and Thomas Woo, CloudStream: delivering high-quality streaming video through a cloud-based H.264/SVC proxy. In Proceedings of IEEE International Conference on Computer Communications (INFOCOM) miniconference, Apr. 2011.

[6] J.-C. Wu, P. Huang, J. J. Yao, and H. H. Chen, A collaborative transcoding strategy for live broadcasting over peer-to-peer IPTV networks. IEEE Trans. Circuits Syst. Video Technol., vol. 21, no. 2, pp. 220-224, Feb. 2011.

[7] D. Jurca and P. Frossard, Media flow rate allocation in multipath networks. IEEE Transactions on Multimedia, vol. 9, no. 6, 2007.

[8] R. Kumar, Y. Liu, and K. W. Ross, Stochastic Fluid Theory for P2P Streaming Systems. In Proc. of IEEE INFOCOM, 2007.

[9] S. Liu, R. Z. Shen, W. Jiang, J. Rexford, and M. Chiang, Performance Bounds for Peer-Assisted Live Streaming. In Proc. of ACM SIGMETRICS, 2008.

[10] C. Feng and B. Li, On large-scale peer-to-peer streaming systems with network coding. In Proc. of the 16th ACM international conference on Multimedia, Vancouver, British Columbia, Canada, 2008, pp. 269-278.

[11] C. Zhao, X. Lin, and C Wu, The streaming capacity of sparsely-connected P2P systems with distributed control. In Proc. of IEEE INFOCOM, pp. 1449-1457, 2011.

[12] S. Sengupta, S. Liu, M. Chen, M. Chiang, J. Li, and P.A. Chou, Peer-to-Peer Streaming Capacity. IEEE Transactions on Information Theory, vol. 57, no. 8, pp. 5072-5087, Aug. 2011. 